\title{
How do health care professionals assess patients when initiating insulin therapy? A qualitative study
}

\begin{abstract}
Aims: To explore how health care professionals (HCPs) assess patients when initiating insulin therapy in type 2 diabetes.

Methods: Focus group discussions and in-depth interviews were conducted with 41 health care professionals in Malaysia in 2010ï 2011. A semi-structured topic guide was used for the interview. The interviews were transcribed verbatim and analysed using the Nvivo9 software based on a thematic approach.

Results: HCPs were less likely to initiate insulin therapy in patients who were older, with irregular dietary patterns and poor financial status. They also assessed patientsôknowledge, views and misconceptions of insulin. However, there was a variation in how doctors assessed patientsôcomorbidities before starting insulin therapy. Medical officers were more likely to initiate insulin therapy in patients with comorbidities and complications, whereas family medicine specialists were more cautious. In addition, most HCPs considered patientsô psychosocial status, including self-care ability, social support and quality of life.

Conclusions: HCPsôassessment of patientsôneed to start insulin therapy depends on their perception rather than objective evaluation of patientsô background, knowledge, perception and abilities. The background and the type of practice of HCPs influence their assessment.
\end{abstract}

Keyword: Physiciansômedical decision; Type 2 diabetes; Insulin therapy initiation 